\title{
On the highest space in which a non-ruled surface of given order can lie
}

By G. Timms.

(Received 8th November, 1939. Read 25th November, 1939.)

It is well known ${ }^{1}$ that a non-ruled (i.e. not consisting of an infinity of lines) surface of order $n$ lies in a space of not more than $n$ dimensions $(n \neq 4)$, and that for $n>9$, the maximum dimension actually attained (here denoted by $R$ ) is certainly less than $n$.

The precise value of $R$ does not seem to have been stated explicitly: the purpose of this note is to shew that

$$
R=I\left(\frac{3}{4} n\right)+2, \quad n \neq 1,2,3,9
$$

where $I(x)$ denotes the integral part of $x$.

This conclusion is deduced from del Re's theorem ${ }^{2}$ that for a nonruled surface whose prime sections are of genus $p(\geqq 2)$, the maximum order is $4 p+4$.

Consider a surface of order $n$ in space of $r$ dimensions, whose prime sections are of genus $p(\geqq 2)$. The series cut on any prime section by all the other prime sections (characteristic series of prime sections) is evidently of order $n$ and freedom $r-1:$ suppose that it is contained in a complete series of freedom $r_{0}-1$ (so that $r \leqq r_{0}$ ), and that its index of speciality is $i$.

From the Riemann-Roch theorem $n-r_{0}+1=p-i$.

From del Re's result quoted above $p \geqq \frac{1}{4} n-1$.

$$
\text { Eliminating } p \quad r_{0} \leqq \frac{3}{4} n+2+i \text {. }
$$

In general $i=0$, i.e. the series is not special, so that

$$
r \leqq r_{0} \leqq \frac{3}{4} n+2 \text {. }
$$

If the series is special, the prime sections are not hyperelliptic (for the characteristic series of a prime section is cut thereon by primes, and is therefore certainly simple). Hence from Clifford's theorem

so that

$$
\begin{gathered}
r_{0}-1 \leqq \frac{1}{2} n, \\
r \leqq r_{0} \leqq \frac{1}{2} n+1<\frac{3}{4} n+2 .
\end{gathered}
$$

Thus in no case for which $p \geqq 2$ can $r$ exceed $I\left(\frac{3}{4} n\right)+2$.

I Del Pezzo, Rend. R. Acc. Napoli, 24 (1885), 215, and 25 (1886), 208.

2 Rend. R. Acc. Napoli (3), 30 (1924), 80. 
The value $r=I\left(\frac{3}{4} n\right)+2$ is attained ${ }^{1}$ by the surface represented upon a plane by curves of order $m$ having one fixed $\overline{m-2}$ fold point and $4 m-4-n$ fixed simple points, $m$ being the least integer $\geqq \frac{1}{4} n+1$. This confirms incidentally del Pezzo's surmise that the maximum $R$ is attained by a rational surface.

It follows that for $p \geqq 2, R=I\left(\frac{3}{4} n\right)+2$.

The cases $p=0,1$ (and therefore $n \leqq 9$ ) are well-known and yield the same value for $R$, except when $n=1,2$ (when non-ruled surfaces are impossible); $n=3,(R=3) ; n=9,(R=9)$.

1 The prime sections are evidently hyperelliptic. The same surfaces, when $1 n$ is an integer, are in fact those haviog the maximum order for a given genus of section.

The University, St AndRews. 